\title{
Estudio de minimización de costes de panitumumab frente a cetuximab en combinación con quimioterapia en primera línea y segunda línea en el cáncer colorrectal metastásico KRAS nativo en España
}

\author{
Cristina Grávalos Castro • Ferran Pérez-Alcántara • \\ José Antonio Gasquet Espuña $\cdot$ Ignasi Campos Tapias
}

Published online: 23 November 2013

(C) Springer International Publishing Switzerland 2013

Resumen Objetivo La evidencia científica indica similar eficacia y tolerancia de los anticuerpos anti-EGFR panitumumab (Pmab) y cetuximab (Cmab). El objetivo de este estudio es la evaluación económica de Pmab frente a Cmab con quimioterapia (QT) en $1^{\mathrm{a}} / 2^{\mathrm{a}}$ línea (L) en el cáncer colorrectal metastásico (CCRm) KRAS nativo en España.

Métodos Análisis de minimización de costes para comparar Pmab+FOLFOX4 frente a Cmab+FOLFOX4/FOLFIRI en $1^{\mathrm{a}} \mathrm{L}$ y Pmab+FOLFIRI frente a Cmab+FOLFOX4/ FOLFIRI en $2^{\mathrm{a}} \mathrm{L}$. Pauta de administración y coste farmacológico (coste por $\mathrm{mg}$, según las fichas técnicas de Pmab/Cmab y citostáticos). Perspectiva del análisis: hospital, considerando costes médicos directos (farmacológicos, administración y manejo del paciente). Horizonte temporal en base a la mediana de ciclos administrados (estudios pivotales de Pmab): $22 / 18$ semanas en $1^{\mathrm{a}} / 2^{\mathrm{a}} \mathrm{L}$, respectivamente. Paciente tipo utilizado: $69,9 \mathrm{~kg} ; 165,4 \mathrm{~cm} ; 1,79 \mathrm{~m}^{2}$. Se realizaron análisis de sensibilidad para evaluar la robustez de los resultados.

C. Grávalos Castro

Servicio de Oncología Médica, Hospital Universitario 12 de Octubre, Madrid, España

\section{F. Pérez-Alcántara $(\varangle)$}

Oblikue Consulting, S.L. Barcelona, c/Josep Irla i Bosch, 5-7, $1^{\text {a }}$ Planta, 08034 Barcelona, España

e-mail: ferran.perez@oblikue.com

J.A. Gasquet Espuña

Departamento médico, AMGEN SA, WTC Barcelona, Moll de Barcelona s/n. Edifici Sud, $7^{\text {a }}$ Planta, 08039 Barcelona, España

I. Campos Tapias

Unidad de Farmacoeconomía e Investigación de Resultados en Salud, AMGEN, SA, WTC Barcelona, Moll de Barcelona s/n. Edifici Sud, 7 a Planta, 08039 Barcelona, España
Resultados El coste del tratamiento con Pmab+FOLFOX4 en $1^{\mathrm{a}} \mathrm{L}(26.074 €)$ fue menor que el de Cmab+FOLFOX4/ FOLFIRI $(31.496 €[17,2 \%]$ y $27.767 €[6,1 \%]$, respectivamente). En $2^{\mathrm{a}} \mathrm{L}, \mathrm{Pmab}+\mathrm{FOLFIRI}$ también produjo menor coste (18.349 €) en comparación con Cmab+FOLFOX4/ FOLFIRI $(25.926 €[29,2 \%]$ y $22.875 €[19,8 \%]$, respectivamente). Analizando únicamente el coste de los anticuerpos, Pmab representaría un ahorro de $1.847 €(9,9 \%)$ y de $1.601 €(10,4 \%)$ en $1^{\mathrm{a}} \mathrm{L}$ y $2^{\mathrm{a}} \mathrm{L}$, respectivamente, comparado con Cmab. Los parámetros claves por su mayor variabilidad e influencia en el análisis fueron peso y altura. El análisis de sensibilidad sobre estas variables mostró que los resultados del modelo fueron robustos.

Conclusiones La combinación de Pmab/QT supondría un ahorro para el Sistema Público de Salud en comparación con Cmab/QT, tanto en $1^{\mathrm{a}} \mathrm{L}$ como en $2^{\mathrm{a}} \mathrm{L}$, en pacientes con CCRm KRAS nativo.

Palabras clave Cáncer colorrectal metastásico . Anticuerpos monoclonales anti-EGFR - Análisis de minimización de costes $\cdot$ España

Abstract Objective Available literature shows a similar efficacy and tolerability profile for anti-EGFR MAb panitumumab (Pmab) and cetuximab (Cmab). The aim of the analysis was to compare the cost of Pmab versus Cmab in 1st and 2nd line settings in combination with chemotherapy (CT) for the treatment of wild-type KRAS metastatic colorectal cancer (mCRC) patients in Spain.

Methods A cost minimization analysis comparing Pmab+ FOLFOX4 versus Cmab+FOLFOX4/FOLFIRI in 1st line and Pmab+FOLFIRI versus Cmab+FOLFOX4/FOLFIRI in 2nd line setting. Dosage and drug cost (cost per mg, based on summary of product characteristics for Pmab/Cmab and cytostatic drugs). Hospital perspective with only direct costs 
was considered (drug, administration and patient management costs). Time horizon based on median number of administered cycles (Pmab pivotal trials): 22/18 weeks for 1st/2nd line, respectively. Patient type considered: $69.9 \mathrm{~kg}$; $165.4 \mathrm{~cm} ; 1.79 \mathrm{~m}^{2}$. Sensitivity analyses were conducted to test the robustness of the analysis.

Results Treatment cost for Pmab+FOLFOX4 in 1st line setting $(26,074 €)$ was lower compared to Cmab+FOLFOX4/ FOLFIRI $(31,496 €[17.2 \%]$ and $27,767 €[6.1 \%]$, respectively). In 2nd line setting, Pmab+FOLFIRI resulted less costly $(18,349 €)$ versus Cmab+FOLFOX4/FOLFIRI $(25,926 €[29.2 \%]$ and $22,875 €[19.8 \%]$, respectively). Furthermore, taking into account the cost of anti-EGFR only, Pmab can be $1,847 €(9.9 \%)$ and $1,601 €(10.4 \%)$ less costly in 1st and 2nd line setting, respectively, compared to Cmab. Key inputs due to their wider variability and influence were weight and height. Sensitivity analysis confirmed the robustness of the results.

Conclusions Pmab/CT regimens may be cost-saving for the National Health Service compared to Cmab/CT regimens in both 1st and 2nd line wild-type KRAS mCRC patients.

Keywords Metastatic colorectal cancer - Anti-EGFR monoclonal antibodies $\cdot$ Cost minimization analysis - Spain

\section{Introducción}

El cáncer colorrectal (CCR) se encuentra entre las primeras localizaciones tumorales en España, con una tasa de incidencia mayor en hombres que en mujeres, con cifras de 66 por cada 100.000 habitantes/año y 34 por cada 100.000 habitantes/año, respectivamente [1]. De los pacientes afectados por CCR, se estima que aproximadamente un $40 \%$ desarrollará un CCR metastásico (CCRm) [2].

En España, la mortalidad por CCR para ambos sexos es la segunda más elevada por tipo de cáncer, después del cáncer de pulmón, y causa cada año 13.200 muertes [1]. De nuevo, los hombres son el sexo más afectado, con una tasa de mortalidad por cada 100.000 habitantes/año de 28 , a comparar con la de 12 muertes por cada 100.000 habitantes/año para las mujeres [1].

La mayor incidencia de un cáncer suele estar relacionada con unos mayores costes para el Sistema Nacional de Salud (SNS) y, por tanto, con una mayor destinación de recursos, tal y como sucede con el CCRm [3]. Por este motivo, es importante estudiar las terapias que consigan minimizar los costes para el SNS.

En las principales guías clínicas nacionales e internacionales $[2,4-6]$ se prioriza la aproximación individualizada del paciente para obtener una mejor respuesta en el tratamiento del CCRm. En este sentido, está aumentando la importancia de los fármacos biológicos dirigidos específicamente a biomarcadores o dianas moleculares.
Se ha demostrado que la vía de señalización del receptor del factor de crecimiento epidérmico (EGFR) desempeña un papel destacado en la patogenia del CCR. La interacción del factor de crecimiento epidérmico (EGF) o el factor de crecimiento transformador (TGF)-alfa con el EGFR conduce a la fosforilación y activación de las tirosina-quinasas que regulan la transcripción de moléculas (incluida la vía RAS-MAPK) que participan en el crecimiento, diferenciación, supervivencia, inhibición de la apoptosis, migración, proliferación y transformación celulares [7]. El EGFR está sobreexpresado con frecuencia en el CCR [8]. Este hecho se asociaría a una progresión más rápida de la enfermedad que posiblemente daría lugar a peores tasas de supervivencia [810]. El gen KRAS nativo ha sido identificado como factor predictivo negativo de respuesta a los anticuerpos monoclonales anti-EGFR (anti-EGFR) lo cual permite identificar a los pacientes con CCRm en los que su uso comporta una relación beneficio/riesgo favorable [11, 12]. Para el caso español, se estima que el 54,1\% de pacientes con CCRm tienen el gen KRAS nativo [13]. La adición de las terapias basadas en anti-EGFR a los regímenes de quimioterapia clásicos ha aumentado la eficacia en el tratamiento del CCRm, y ha conseguido mejorar tanto las tasas de supervivencia global, supervivencia libre de progresión o las tasas de respuesta [14-17] como la calidad de vida de los pacientes [18].

Cetuximab (Erbitux ${ }^{\circledR}$ ) y panitumumab $\left(\right.$ Vectibix $^{\circledR}$ ) son anti-EGFR, de composición quimérica el primero y completamente humano el segundo, que actúan uniéndose al dominio externo del EGFR, y compiten con sus ligandos naturales. La eficacia y seguridad de panitumumab y de cetuximab han sido demostradas en diversos estudios tanto en primera como en segunda línea de tratamiento del CCRm [19-22], como ya había sido demostrado con anterioridad en tercera línea de tratamiento [23]. La evidencia actualmente existente parece indicar una eficacia similar de ambos, tanto en monoterapia, en la que existe un estudio comparativo directo, como en combinación con quimioterapia convencional [19-21, 23-28].

El objetivo del presente estudio ha sido comparar el coste de tratamiento del CCRm en primera y segunda línea de tratamiento en pacientes con CCRm y KRAS nativo con panitumumab y cetuximab, ambos en combinación con los regímenes habituales de quimioterapia según las correspondientes indicaciones.

\section{Métodos}

Tipo de análisis

La evidencia científica disponible indica una similar eficacia de panitumumab y cetuximab como tratamiento del CCRm en pacientes con el gen KRAS nativo [26-28]. Es por ello 
Tabla 1 Resumen de asunciones para la estimación del coste farmacológico de los anti-EGFR y según línea de tratamiento

\begin{tabular}{|c|c|c|c|c|c|c|}
\hline \multirow[t]{2}{*}{ Tipo de administración } & \multicolumn{2}{|l|}{ Panitumumab } & \multicolumn{4}{|l|}{ Cetuximab } \\
\hline & \multicolumn{2}{|c|}{ IV 1 hora infusión } & \multicolumn{2}{|c|}{ IV 2 horas infusión } & \multicolumn{2}{|c|}{ IV 1 hora infusión } \\
\hline 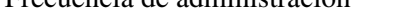 & \multicolumn{2}{|l|}{ Bisemanal } & \multicolumn{4}{|l|}{ Semanal } \\
\hline $\begin{array}{l}\text { Número de administraciones en } 1^{\mathrm{a}} \\
\text { línea }\end{array}$ & \multicolumn{2}{|l|}{11} & \multicolumn{4}{|l|}{22} \\
\hline \multirow{3}{*}{$\begin{array}{l}\text { Número de administraciones en } 2^{\mathrm{a}} \\
\text { línea }\end{array}$} & \multicolumn{2}{|l|}{9} & \multicolumn{4}{|l|}{18} \\
\hline & \multirow[t]{2}{*}{ Dosis $(\mathrm{mg} / \mathrm{kg})$} & \multirow[t]{2}{*}{ Dosis total (mg) } & \multicolumn{2}{|l|}{ Dosis inicial } & \multicolumn{2}{|c|}{ Dosis posteriores } \\
\hline & & & Dosis $\left(\mathrm{mg} / \mathrm{m}^{2}\right)$ & Dosis total (mg) & Dosis $\left(\mathrm{mg} / \mathrm{m}^{2}\right)$ & Dosis total (mg) \\
\hline Dosis por administración & 6 & 419,40 & 400 & 716,83 & 250 & 448,02 \\
\hline Coste por $\mathrm{mg}^{*}$ & \multicolumn{2}{|c|}{$3,660 €$} & \multicolumn{4}{|c|}{$1,850 €$} \\
\hline
\end{tabular}

\section{IV: Intravenoso}

* PVL más IVA aplicando el descuento del 7,5\% según Real Decreto de Ley 8/2010 [35]

que se ha llevado a cabo un análisis de minimización de costes como método de estudio más apropiado para comparar económicamente ambas opciones.

Opciones de comparación

El análisis comparó las diferentes combinaciones de panitumumab y cetuximab con quimioterapia convencional según las correspondientes indicaciones en ficha técnica para el tratamiento del CCRm en primera y segunda línea [29, 30].

Como primera línea de tratamiento del CCRm se compararon las siguientes alternativas:

- Panitumumab en combinación con FOLFOX4

- Cetuximab en combinación con FOLFOX4

- Cetuximab en combinación con FOLFIRI

Como segunda línea de tratamiento del CCRm se compararon las siguientes alternativas:

- Panitumumab en combinación con FOLFIRI

- Cetuximab en combinación con FOLFOX4

- Cetuximab en combinación con FOLFIRI

\section{Perspectiva y horizonte temporal}

El análisis se llevó a cabo desde la perspectiva del hospital, considerando de forma exclusiva los costes directos sanitarios. Para establecer el horizonte temporal del presente análisis se ha utilizado la mediana de ciclos administrados de panitumumab indicados en los estudios pivotales de panitumumab, 11 en primera línea de tratamiento y 9 en segunda $[19,20]$, que corresponden a 22 y 18 semanas respectivamente, dado que los ciclos de administración de este fármaco son de cada dos semanas, a diferencia de cetuximab con el que los ciclos son semanales. No se aplicó tasa de descuento alguna, ya que el horizonte temporal del análisis fue inferior a un año. Todos los costes fueron expresados en euros del año 2012.

Uso de recursos y costes

El modelo construido para llevar a cabo el análisis, combinó datos sobre el uso de recursos sanitarios en el tratamiento de los pacientes con CCRm y gen KRAS nativo, concretamente datos relativos a los costes farmacológicos (tanto del anti-EGFR como de la quimioterapia) y costes de manejo de los pacientes. La información sobre el uso de recursos así como las asunciones del análisis se obtuvieron a partir de bibliografía relacionada y validada en base a la experiencia clínica.

En primer lugar, el coste farmacológico de las diferentes alternativas de tratamiento viene dado por el coste de los anti-EGFR y el coste de los regímenes de quimioterapia administrados en combinación. La pauta de administración según línea de tratamiento se obtuvo a partir de la dosis recomendada en las correspondientes fichas técnicas $[29,30]$ y los correspondientes ensayos pivotales [19-22] (Tablas 1 y 2). Las características antropométricas consideradas para estimar la dosis por administración se obtuvieron de un estudio español [31] a partir de la base de datos LifeLink $^{\mathrm{TM}}$ Oncology Analyzer ${ }^{\mathrm{TM}}$ de IMS Consulting Group, que indicó un peso corporal medio de $69,9 \mathrm{~kg}$ (desviación estándar 11,5) y una talla media de $165,4 \mathrm{~cm}$ (desviación estándar 8,5) para el paciente tipo con CCRm en España. La superficie corporal media calculada fue de $1,79 \mathrm{~m}^{2}$ aplicando la fórmula de Mosteller [32, 33]. El coste farmacológico se estimó a partir de los precios unitarios públicos para España disponibles en el Consejo Gene- 
Tabla 2 Resumen de asunciones para la estimación del coste farmacológico por régimen de quimioterapia y según línea de tratamiento

\begin{tabular}{|c|c|c|c|c|c|}
\hline & & \multicolumn{2}{|c|}{ FOLFIRI } & \multicolumn{2}{|c|}{ FOLFOX4 } \\
\hline \multicolumn{3}{|l|}{ Tipo de administración } & \multicolumn{2}{|c|}{ IV infusión 1 día } & IV infusión 2 días \\
\hline \multicolumn{3}{|c|}{ Frecuencia de la administración } & \multicolumn{2}{|c|}{ Bisemanal } & Bisemanal \\
\hline \multicolumn{3}{|c|}{ Número de administraciones en $1^{\mathrm{a}}$ línea } & \multicolumn{2}{|c|}{11} & 11 \\
\hline \multicolumn{2}{|c|}{ Número de administraciones en $2^{\mathrm{a}}$ línea } & \multicolumn{2}{|c|}{9} & \multicolumn{2}{|c|}{9} \\
\hline & \multirow[b]{2}{*}{$\begin{array}{l}\text { Coste por } \\
\mathrm{mg}^{*}\end{array}$} & \multicolumn{2}{|c|}{ Dosis por administración } & \multicolumn{2}{|c|}{ Dosis por administración } \\
\hline & & $\begin{array}{l}\text { Dosis } \\
\left(\mathrm{mg} / \mathrm{m}^{2}\right)\end{array}$ & $\begin{array}{l}\text { Dosis total } \\
(\mathrm{mg})\end{array}$ & $\begin{array}{l}\text { Dosis } \\
\left(\mathrm{mg} / \mathrm{m}^{2}\right)\end{array}$ & $\begin{array}{l}\text { Dosis total } \\
(\mathrm{mg})\end{array}$ \\
\hline Irinotecán & $0,224 €$ & $180 \mathrm{~d} 1$ & 322,57 & - & - \\
\hline Oxaliplatino & $1,323 €$ & - & - & $85 \mathrm{~d} 1$ & 152,33 \\
\hline Fluorouracilo bolus & $0,004 €$ & $400 \mathrm{~d} 1$ & 716,83 & $400 \mathrm{~d} 1-2$ & $1.433,66$ \\
\hline Fluorouracilo infusión & $0,004 €$ & $2.400 \mathrm{~d} 1$ & $4.300,97$ & $600 \mathrm{~d} 1-2$ & $2.150,48$ \\
\hline Leucovorín & $0,063 €$ & $400 \mathrm{~d} 1$ & 716,83 & $200 \mathrm{~d} 1-2$ & 716,83 \\
\hline
\end{tabular}

IV: Intravenoso

* PVL más IVA

d1: Día 1 del ciclo; d1-2: Días 1 y 2 del ciclo

ral de Colegios Oficiales de Farmacéuticos expresados como precios de venta del laboratorio (PVL) [34], considerando el IVA correspondiente y asumiendo la reutilización del vial. El coste farmacológico estimado también consideró los posibles descuentos asociados al Real Decreto de Ley 8/2010 [35].

En cuanto a los costes relacionados con el manejo de los pacientes, se consideraron las visitas del oncólogo, las analíticas sanguíneas, la realización de la determinación del estado del gen KRAS (únicamente para la primera visita) y la visita en el hospital de día oncológico para la administración de los fármacos (Tabla 3). Para el segundo día del ciclo para la administración de fluorouracilo y leucovorín en el esquema FOLFOX4, únicamente se consideró el coste de la visita en el hospital de día oncológico. El coste unitario de los recursos utilizados en el manejo de los pacientes se obtuvo de la base de datos de costes sanitarios eSalud [36], a excepción del coste de la determinación KRAS que se estimó a partir de datos internos de Amgen.

\section{Análisis de sensibilidad}

Con el fin de valorar la influencia de la incertidumbre de algunos parámetros incluidos en el análisis y validar así la robustez de los resultados obtenidos, se utilizaron técnicas de análisis de sensibilidad.

Los parámetros identificados como clave debido a su mayor variabilidad e influencia en el análisis fueron el peso y la altura, debido a que de ellos depende la dosis total administrada al paciente. Este hecho es especialmente relevante para el presente análisis ya que, mientras que la dosis de
Tabla 3 Resumen de asunciones para la estimación de los costes relacionados con el manejo de los pacientes

\begin{tabular}{lll}
\hline & Coste & Fuente \\
\hline Coste primera visita oncología (a) & $161,39 €$ & eSalud [36] \\
Coste siguientes visitas oncología (b) & $96,83 €$ & eSalud [36] \\
Análisis sanguíneo (c) & $12,53 €$ & eSalud [36] \\
Hospital de día oncológico (d) & $215,65 €$ & eSalud [36] \\
Determinación KRAS (e) & $299,40 €$ & Datos internos \\
& & Amgen \\
Coste primera administración & $\mathbf{6 8 9} €$ & $\mathbf{a}+\mathbf{c}+\mathbf{d}+\mathbf{e}$ \\
Coste siguientes administraciones d1 & $\mathbf{3 2 6} €$ & $\mathbf{a + b}+\mathbf{c}+\mathbf{d}$ \\
Coste siguientes administraciones d2 & $\mathbf{2 1 6} €$ & $\mathbf{d}$ \\
\hline
\end{tabular}

d1: Día 1 del ciclo; d2: Día 2 del ciclo

panitumumab depende del peso, la dosis de cetuximab depende de la superficie corporal del paciente [29]. Para su evaluación, se llevó a cabo un análisis de sensibilidad probabilístico mediante la técnica de Monte-Carlo de segundo orden, siguiendo las recomendaciones internacionales más relevantes sobre el análisis de la incertidumbre de los estudios de evaluación económica en el ámbito sanitario [3739]. Los resultados del caso base se simularon sobre una cohorte hipotética de 1.000 pacientes con el fin de observar la probabilidad con la que los resultados se mantienen estables ante la variación del peso y la altura de los pacientes. Tal variación se introdujo asignando una distribución normal para el peso y la altura a partir de la desviación estándar correspondiente. 
Tabla 4 Resultados de minimización de costes en $1^{\mathrm{a}}$ línea

\begin{tabular}{|c|c|c|c|c|c|}
\hline $\begin{array}{l}\text { Coste total del } \\
\text { tratamiento }\end{array}$ & $\mathrm{PMab}+\mathrm{FOLFOX} 4^{\mathrm{a}}$ & $\mathrm{CMab}+\mathrm{FOLFIRI}^{\mathrm{b}}$ & $\begin{array}{l}\text { PMab+FOLFOX4 vs } \\
\text { CMab+FOLFIRI }\end{array}$ & $\mathrm{CMab}+\mathrm{FOLFOX} 4^{\mathrm{b}}$ & $\begin{array}{l}\text { PMab+FOLFOX4 vs } \\
\text { CMab+FOLFOX4 }\end{array}$ \\
\hline $\begin{array}{l}\text { Coste farmacológico } \\
\text { anti-EGFR }\end{array}$ & $16.885 €$ & $18.732 €$ & $\begin{array}{l}-1.847 € \\
(-10 \%)\end{array}$ & $18.732 €$ & $\begin{array}{l}-1.847 € \\
(-10 \%)\end{array}$ \\
\hline $\begin{array}{l}\text { Coste farmacológico } \\
\text { quimioterapia }\end{array}$ & $2.878 €$ & $1.521 €$ & $\begin{array}{l}1.356 € \\
(89 \%)\end{array}$ & $2.878 €$ & $\begin{array}{l}0 € \\
(0 \%)\end{array}$ \\
\hline Coste administración & $4.744 €$ & $4.744 €$ & $\begin{array}{l}0 € \\
(0 \%)\end{array}$ & $7.116 €$ & $\begin{array}{l}-2.372 € \\
(-33 \%)\end{array}$ \\
\hline $\begin{array}{l}\text { Otros costes de } \\
\text { manejo }^{\mathrm{d}}\end{array}$ & $1.567 €$ & $2.770 €$ & $\begin{array}{l}-1.203 € \\
(-43 \%)\end{array}$ & $2.770 €$ & $\begin{array}{l}-1.203 € \\
(-43 \%)\end{array}$ \\
\hline Costes totales & $26.074 € €$ & $27.767 €$ & $\begin{array}{l}-\mathbf{1 . 6 9 3} € \\
(-6 \%)\end{array}$ & $31.496 €$ & $\begin{array}{l}-\mathbf{5 . 4 2 2} € \\
(-17 \%)\end{array}$ \\
\hline
\end{tabular}

a PMab: Panitumumab: 11 administraciones en $1^{\mathrm{a}}$ línea

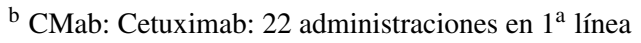

c Anti-EGFR: Anticuerpos monoclonales inhibidores del receptor del factor de crecimiento epidérmico

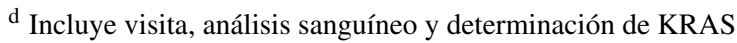

Tabla 5 Resultados de minimización de costes en $2^{\mathrm{a}}$ línea

\begin{tabular}{|c|c|c|c|c|c|}
\hline $\begin{array}{l}\text { Coste total del } \\
\text { tratamiento }\end{array}$ & PMab+FOLFIRI & CMab+FOLFIRI ${ }^{b}$ & $\begin{array}{l}\text { PMab+FOLFIRI vs } \\
\text { CMab+FOLFIRI }\end{array}$ & $\mathrm{CMab}+\mathrm{FOLFOX} 4^{\mathrm{b}}$ & $\begin{array}{l}\text { PMab+FOLFIRI vs } \\
\text { CMab+FOLFOX4 }\end{array}$ \\
\hline $\begin{array}{l}\text { Coste farmacológico } \\
\text { anti-EGFR }^{\mathrm{c}}\end{array}$ & $13.815 €$ & $15.416 €$ & $\begin{array}{l}-1.601 € \\
(-10 \%)\end{array}$ & $15.416 €$ & $\begin{array}{l}-1.601 € \\
(-10 \%)\end{array}$ \\
\hline $\begin{array}{l}\text { Coste farmacológico } \\
\text { quimioterapia }\end{array}$ & $1.245 €$ & $1.245 €$ & $\begin{array}{l}0 € \\
(0 \%)\end{array}$ & $2.354 €$ & $\begin{array}{l}-1.110 € \\
(-47 \%)\end{array}$ \\
\hline Coste administración & $1.941 €$ & $3.882 €$ & $\begin{array}{l}-1.941 € \\
(-50 \%)\end{array}$ & $5.823 €$ & $\begin{array}{l}-3.882 € \\
(-67 \%)\end{array}$ \\
\hline $\begin{array}{l}\text { Otros costes de } \\
\text { manejo }^{\mathrm{d}}\end{array}$ & $1.348 €$ & $2.332 €$ & $\begin{array}{l}-984 € \\
(-42 \%)\end{array}$ & $2.332 €$ & $\begin{array}{l}-984 € \\
(-42 \%)\end{array}$ \\
\hline Costes totales & $18.349 €$ & $22.875 €$ & $\begin{array}{l}-4.526 € \\
(-20 \%)\end{array}$ & $25.926 €$ & $\begin{array}{l}-7.577 € € \\
(-29 \%)\end{array}$ \\
\hline
\end{tabular}

${ }^{a}$ PMab: Panitumumab: 9 administraciones en $2^{\mathrm{a}}$ línea

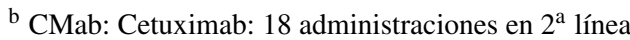

${ }^{c}$ Anti-EGFR: Anticuerpos monoclonales inhibidores del receptor del factor de crecimiento epidérmico

${ }^{\mathrm{d}}$ Incluye visita, análisis sanguíneo y determinación de KRAS

De igual forma, se estimaron los resultados considerando únicamente los costes farmacológicos de los anti-EGFR y llevando a cabo el mismo tipo de análisis de sensibilidad probabilístico sobre el peso y la altura.

Los resultados de los análisis de sensibilidad probabilísticos correspondientes se muestran más adelante en forma de un histograma en el que se agrupan el número de simulaciones según la diferencia de costes observada.

Debido a la relevancia que tienen las características antropométricas en la estimación de los costes, se ha llevado a cabo un análisis de sensibilidad multivariante en el que se han variado de forma simultánea el peso y la altura en función del máximo y mínimo resultantes de las correspondientes desviaciones estándar. Este análisis permite estimar los costes según tipo de paciente y, en concreto, evaluar el impacto en pacientes con un grado variable de obesidad [40].

\section{Resultados}

\section{Resultados básicos}

Los resultados del análisis mostraron que la alternativa de panitumumab en combinación con quimioterapia es menos costosa que cetuximab en combinación con quimioterapia tanto en primera (Tabla 4) como en segunda línea (Tabla 5) de tratamiento de los pacientes con CCRm y KRAS nativo. 
En primera línea de tratamiento, la combinación de panitumumab+FOLFOX 4 produce un menor coste, tanto si se compara con la combinación cetuximab+FOLFOX4 (un ahorro del 17\%) como si se compara con cetuximab+ FOLFIRI (un ahorro del 6\%). En la segunda línea de tratamiento, la combinación de panitumumab+FOLFIRI también produjo un menor coste en comparación con cetuximab+FOLFIRI (un ahorro del 20\%) y con cetuximab +FOLFOX4 (un ahorro del 29\%).

\section{Resultados del análisis de sensibilidad}

Si analizamos únicamente el coste que suponen los antiEGFR, el uso de panitumumab representaría un ahorro de $1.847 €(10 \%)$ en comparación con cetuximab en primera línea de tratamiento y de $1.601 €(10 \%)$ en segunda línea de tratamiento.

El análisis de sensibilidad probabilístico realizado sobre el peso y la altura confirmó la solidez de los resultados obtenidos. La gran mayoría de los 1.000 casos simulados en primera línea mostraron que la combinación de panitumumab+FOLFOX4 comportaba un menor coste tanto en comparación con cetuximab+FOLFIRI (93\%) como en comparación con cetuximab+FOLFOX4 (100\%) (Fig. 1).

Para la segunda línea de tratamiento, el $100 \%$ de los 1.000 casos simulados mostraron que la combinación de panitumumab+FOLFIRI produjo un menor coste que la combinación de cetuximab con quimioterapia (Fig. 2).

Al considerar exclusivamente el coste farmacológico de ambos anti-EGFR, el mismo análisis de sensibilidad probabilístico anterior muestra que, en la mayoría de casos simulados, panitumumab sigue mostrando un menor coste con respecto a cetuximab tanto en primera línea (96\% de las simulaciones) (Fig. 3), como en segunda línea (97\% de las simulaciones) (Fig. 4).

Los resultados del análisis de sensibilidad multivariante en el que se estimaron los costes de cada alternativa en pacientes con diferentes características antropométricas mostraron como, en la mayoría de los casos, panitumumab en combinación con quimioterapia comportó un menor coste en comparación con cetuximab en combinación con quimioterapia (Tabla 6). Según las características antropométricas del individuo, se puede clasificar su estado nutricional según la clasificación de la Organización Mundial de la Salud (OMS) [41]. Se observa como únicamente al comparar el tratamiento de panitumumab+FOLFOX4 frente a cetuximab+FOLFIRI en primera línea en algunos pacientes con sobrepeso u obesidad, panitumumab+FOLFOX4 produjo un mayor coste.

\section{Discusión}

El CCR se considera uno de los tumores que más recursos consume y genera un gran impacto económico en el SNS español [42]. Las conclusiones del presente estudio muestran que panitumumab en combinación con quimioterapia es una opción para el tratamiento del CCRm menos costosa que cetuximab en combinación con quimioterapia, tanto en primera línea como en segunda línea de tratamiento.

El presente análisis considera las características antropométricas de los pacientes con CCRm en España recogidas a partir de una base de datos específica con datos locales [31], los cuales están en línea con los datos de otros estudios llevados a cabo [43, 44]. El cálculo del coste por alternativa consideró los principales usos de recursos relevantes para el SNS, como son el coste farmacológico (anti-EGFR y quimioterapia), el coste de administración y el coste del manejo de los pacientes. Se llevaron a cabo diversos análisis de sensibilidad para validar la robustez de los resultados del estudio, que fueron uniformemente favorables a panitumumab.

Pese a que el presente análisis es uno de los primeros en analizar las terapias indicadas tanto en primera como en segunda línea en pacientes con el gen KRAS nativo, recientemente se han presentado diversas comunicaciones a congresos con análisis similares, aunque su metodología no permite comparar resultados. Un análisis de coste-efectividad [45] en el que se comparó el tratamiento en primera línea de cetuximab y panitumumab se basó en datos de eficacia y asunciones derivadas de estudios con características, objetivos, diseño y poblaciones muy diferentes. Además, las características antropométricas utilizadas para estimar el coste farmacológico por paciente (70 $\mathrm{kg} \mathrm{y} 1,7 \mathrm{~m}^{2}$ ) corresponden a un paciente de $148 \mathrm{~cm}$ de altura, lo cual no es representativo del perfil de paciente con CCRm en España. En un segundo análisis de coste-efectividad [46] que comparó el tratamiento en primera línea en base a factores predictivos de la respuesta, además de utilizar datos de eficacia no comparables al no disponerse de datos para el subgrupo analizado, tampoco las características antropométricas fueron representativas del perfil de paciente con CCRm en España.

Además, se llevó a cabo la comparación con otra alternativa de tratamiento que podría ser relevante. El tratamiento de cetuximab en combinación con irinotecán $\left(180 \mathrm{mg} / \mathrm{m}^{2}\right.$ administrado cada dos semanas) se comparó también con panitumumab+FOLFIRI. El coste medio estimado del tratamiento de cetuximab+irinotecán en segunda línea fue de $22.281 €$, lo que supone un mayor coste de $3.932 €$ en comparación con panitumumab+FOLFIRI [47].

El presente análisis de minimización de costes presenta diversas limitaciones. En primer lugar, cabe recordar que se trata de un modelo teórico que intenta, a partir de realizar una simulación simplificada de la realidad, reproducir los costes reales generados en la práctica clínica. 
Figura 1 Casos acumulados según diferencia de coste para las 1.000 simulaciones del análisis de sensibilidad probabilístico en primera línea (coste total de tratamiento)
Figura 2 Casos acumulados según diferencia de coste para las 1.000 simulaciones del análisis de sensibilidad probabilístico en segunda línea (coste total de tratamiento)
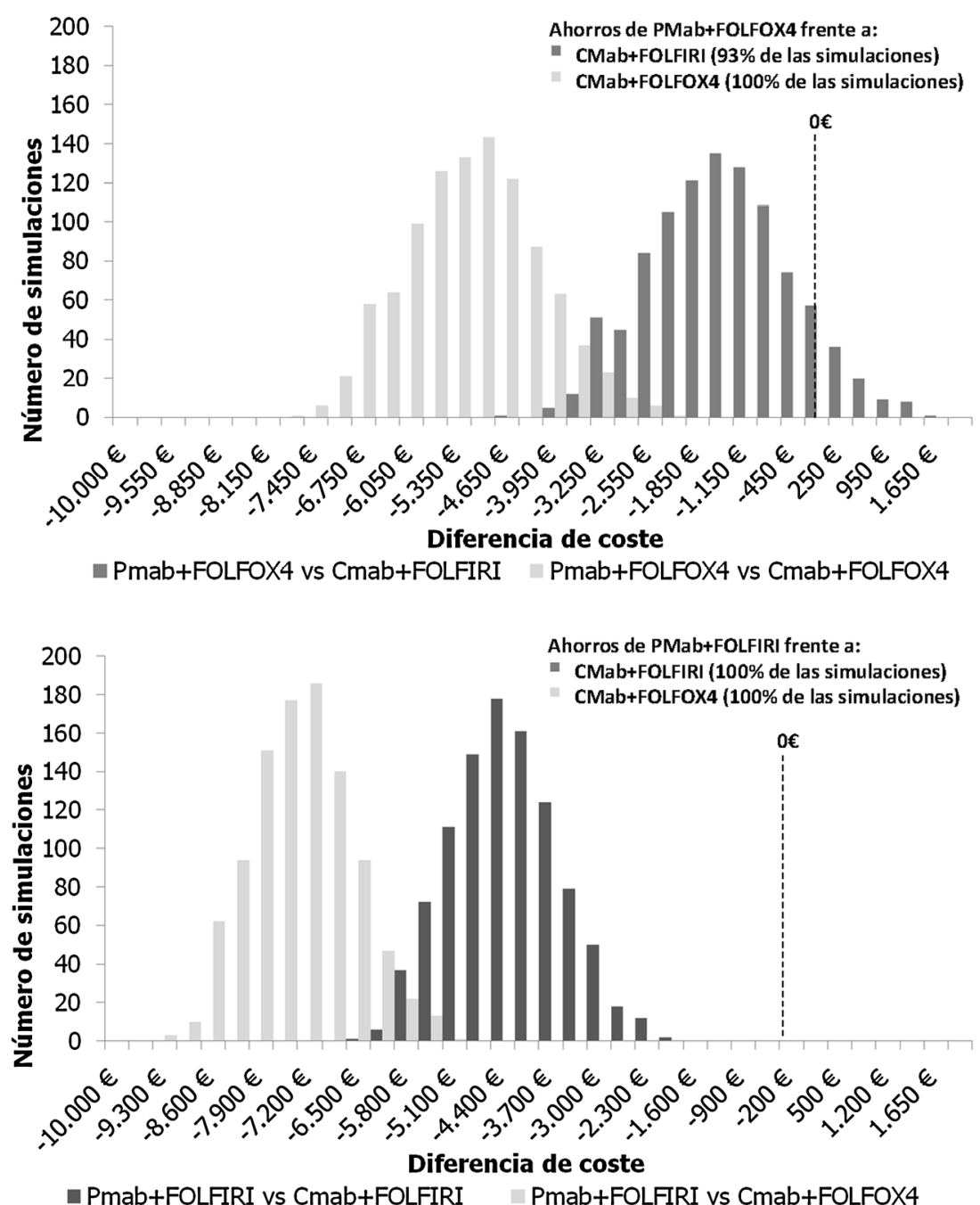

En segundo lugar, cabe mencionar que el presente análisis evalúa las diferencias de coste entre las combinaciones de panitumumab y cetuximab con quimioterapia basándose en que su eficacia es equivalente, pese a que hasta el momento no se dispone de ningún ensayo clínico que compare directamente la eficacia de estas alternativas de tratamiento del CCRm. Sin embargo, la evidencia actualmente disponible parece indicar una eficacia similar de ambas opciones [26] e incluso las conclusiones de un informe técnico del Instituto Catalán de Oncología en el que se comparan ambos tratamientos reafirman este punto [27]. Además, cabe destacar que los resultados de un reciente estudio de fase 3 comparativo de ambos fármacos refuerzan tal asunción, al demostrar la no inferioridad de panitumumab frente a cetuximab de forma directa [28]. Las anteriores evidencias apoyan la metodología empleada en el presente estudio.

Las características antropométricas de los pacientes, como el peso y la altura, se basaron en los pacientes de la base de datos analizada y publicada en 2011 [31]. La influencia de las posibles diferencias de estas variables entre los pacientes de la práctica clínica española se estudió mediante un análisis de sensibilidad tanto probabilístico como multivariante. En el análisis de sensibilidad multivariante, se pudo observar que a mayor índice de masa corporal, el menor coste de la combinación de panitumumab en comparación con la combinación de cetuximab se reduce y únicamente en algunos pacientes con sobrepeso u obesidad tratados en primera línea, las conclusiones del estudio podrían variar.

En el presente análisis no se consideraron otros costes relacionados con la administración de los anti-EGFR, como los costes de premedicación y la monitorización del paciente, costes que podrían ser imputados a cetuximab pero que no son requeridos en el caso de panitumumab. Tampoco se han considerado los costes asociados a los efectos adversos. Los efectos adversos asociados al uso de anti-EGFR, como son panitumumab o cetuximab, son de toxicidad cutánea, diarrea, hipomagnesemia y reacciones infusionales. De todos modos, las reacciones infusionales son menos frecuentes con panitumumab y la razón de ello podría ser su composición $100 \%$ humana [48]. 


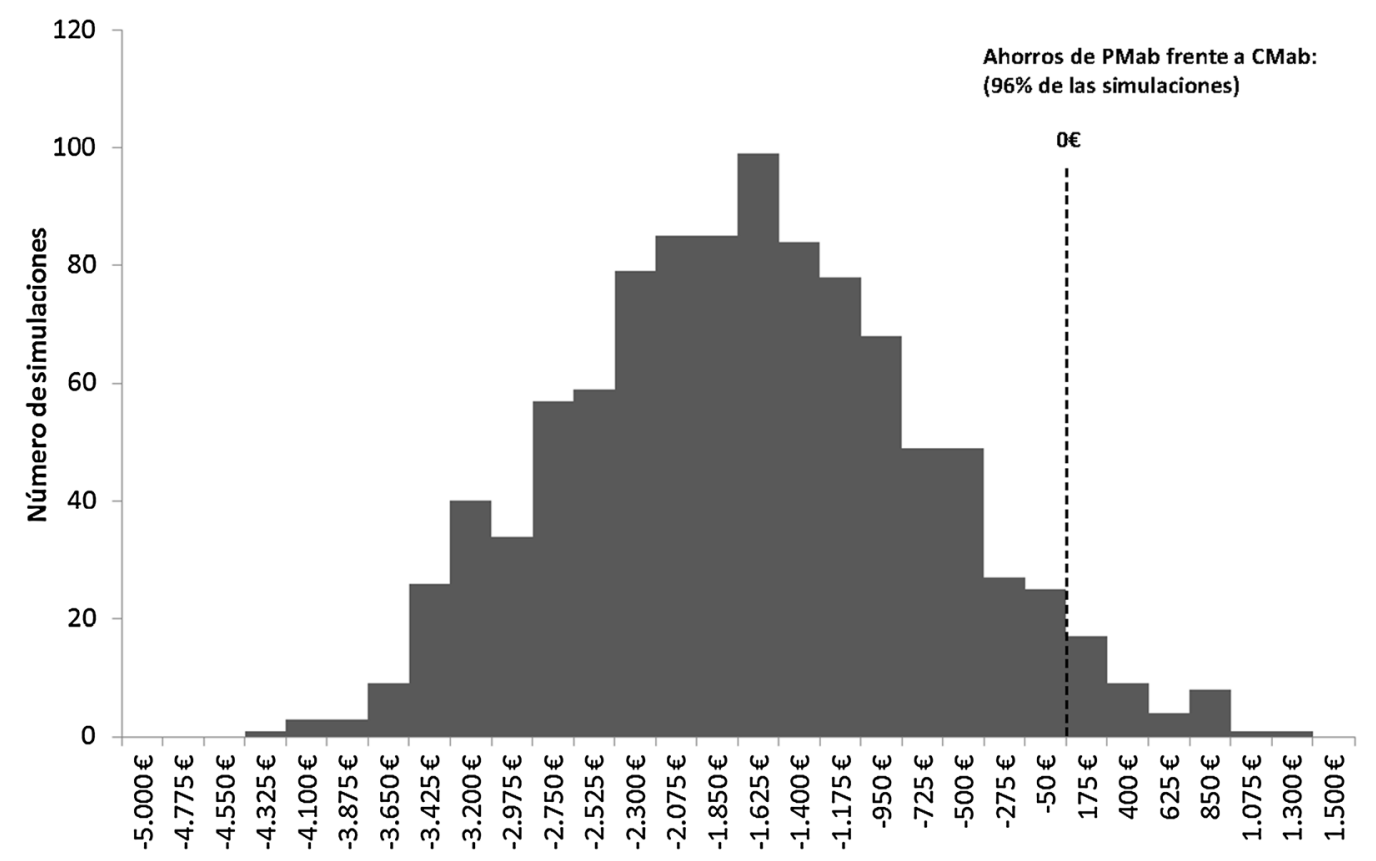

\section{Diferencia de coste}

amab vs $\mathrm{Cmab}$

Figura 3 Casos acumulados según diferencia de coste para las 1.000 simulaciones del análisis de sensibilidad probabilístico en primera línea (coste exclusivamente del anti-EGFR)

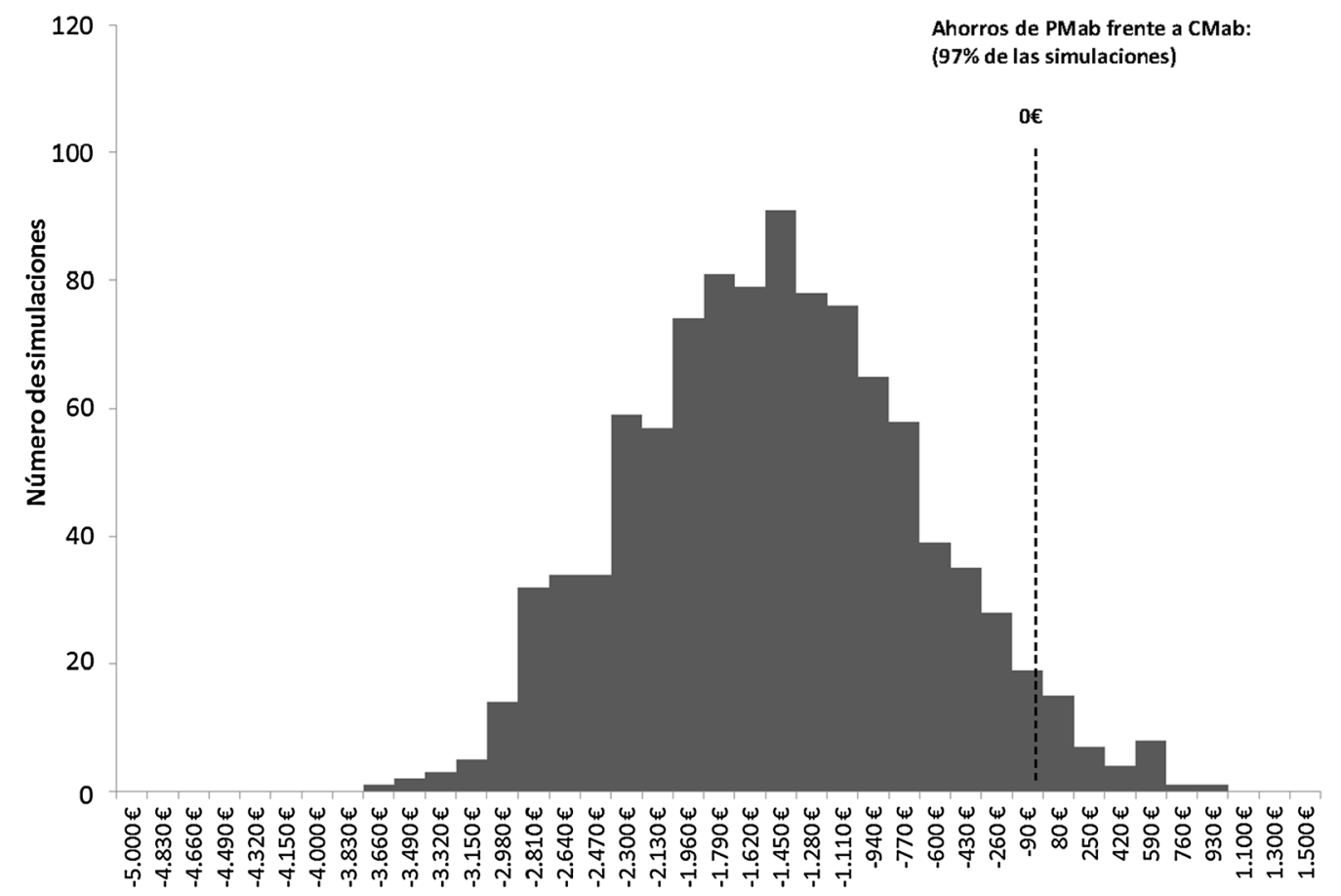

Diferencia de coste

- Pmab vs $\mathrm{Cmab}$

Figura 4 Casos acumulados según diferencia de coste para las 1.000 simulaciones del análisis de sensibilidad probabilístico en segunda línea (coste exclusivamente del anti-EGFR)

\section{A Adis}


Tabla 6 Análisis de sensibilidad multivariante (peso y altura). Resultados de la comparación de costes según clasificación del estado nutricional

Índice de Masa Corporal y estado nutricional OMS (41)

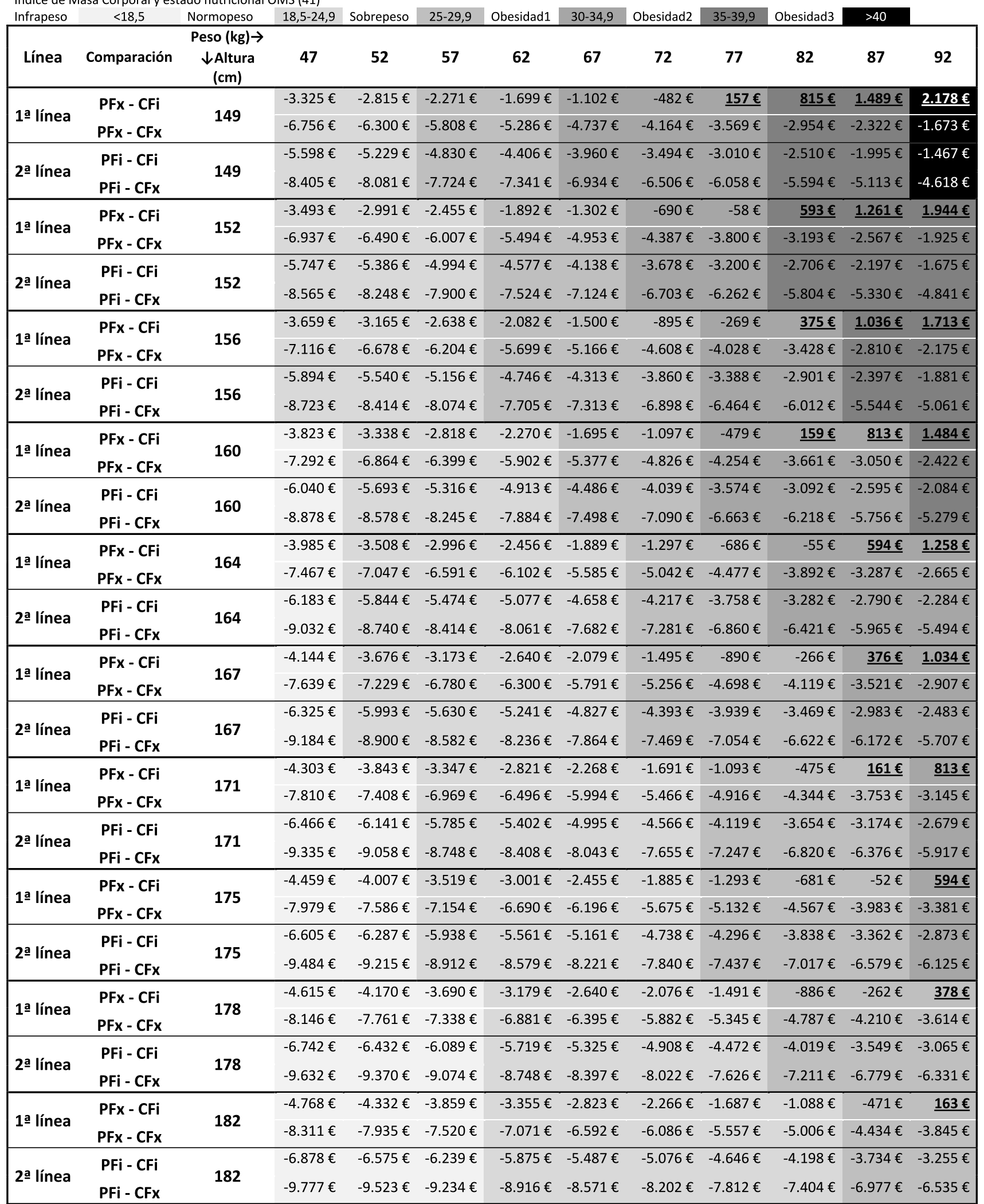

PFx: Panitumumab+FOLFOX4; PFi: Panitumumab+FOLFIRI; CFx: Cetuximab+FOLFOX4; CFi: Cetuximab+FOLFIRI. En negrita y subrayado, resultados en los que la combinación de panitumumab+quimioterapia presentan un mayor coste que cetuximab+quimioterapia. 
A pesar de las anteriores limitaciones del modelo, los distintos análisis de sensibilidad llevados a cabo confirmaron la robustez de los resultados principales.

En conclusión, la evaluación realizada sugiere que la combinación de panitumumab con quimioterapia supondría un ahorro para el SNS en términos de costes directos en comparación con la combinación de cetuximab con quimioterapia tanto en primera línea como en segunda línea de tratamiento de pacientes con CCRm y KRAS nativo.

Conflicto de intereses Cristina Grávalos Castro ha participado como experto clínico en la revisión del manuscrito y declara no tener conflicto de interés en relación con este artículo. José Antonio Gasquet Espuña e Ignasi Campos Tapias trabajan para Amgen que es la empresa promotora del estudio. Ferran Pérez Alcántara trabaja para la consultora Oblikue Consulting que recibió una subvención de Amgen para la realización del estudio.

\section{Bibliografía}

1. Sánchez MJ, Payer T, De Angelis R, et al. Cancer incidence and mortality in Spain: estimates and projections for the period 19812012. Ann Oncol. 2010;21(Suppl 3):iii30-36.

2. García-Carbonero R, Gómez España MA, Casado Sáenz E, et al. SEOM clinical guidelines for the treatment of advanced colorectal cancer. Clin Transl Oncol. 2010;12:729-34.

3. Díez-Fernández R, Girón-Duch C. Treatment costs for metastatic colorectal cancer. Farm Hosp. 2007;31:67-8.

4. Aranda E, Abad A, Carrato A, et al. Treatment recommendations for metastatic colorectal cancer. Clin Transl Oncol. 2011;13:16278.

5. National Comprehensive Cancer Network. Rectal Cancer. V.3.2013. NCCN Clinical Practice Guidelines in Oncology 2013.

6. Schmoll HJ, Van Cutsem E, Stein A, et al. ESMO consensus guidelines for management of patients with colon and rectal cancer. A personalized approach to clinical decision making. Ann Oncol. 2012;23:2479-516.

7. Scaltriti M, Baselga J. The epidermal growth factor receptor pathway: a model for targeted therapy. Clin Cancer Res. 2006;12:5268-72.

8. Spano JP, Lagorce C, Atlan D, et al. Impact of EGFR expression on colorectal cancer patient prognosis and survival. Ann Oncol. 2005; 16:102-8.

9. Kopp R, Rothbauer E, Mueller E, et al. Reduced survival of rectal cancer patients with increased tumor epidermal growth factor receptor levels. Dis Colon Rectum. 2003;46:1391-9.

10. Nicholson RI, Gee JM, Harper ME. EGFR and cancer prognosis. Eur J Cancer. 2001;37(Suppl 4):S9-15.

11. Baselga J, Rosen N. Determinants of RASistance to antiepidermal growth factor receptor agents. J Clin Oncol. 2008;26:1582-4.

12. Berardi R, Onofri A, Pistelli M, et al. Panitumumab: the evidence for its use in the treatment of metastatic colorectal cancer. Core Evid. 2010;5:61-76.

13. García-Foncillas J, Honorato B, Caldés T, et al. "Determina KRAS" project: what's new after twenty months? Ann Oncol. 2010;21(Suppl 8):viii192.

14. Hernández-Losa J, Sanz J, Landolfi S, et al. Recomendaciones para la determinación de mutaciones de K-RAS en cáncer de colon. Rev Esp Patol. 2012;45:76-85.

15. Grothey A. Medical treatment of advanced colorectal cancer in 2009. Ther Adv Med Oncol. 2009;1:55-68.
16. Chibaudel B, Tournigand C, André T, et al. Therapeutic strategy in unresectable metastatic colorectal cancer. Ther Adv Med Oncol. 2012;4:75-89.

17. Edwards MS, Chadda SD, Zhao Z, et al. A systematic review of treatment guidelines for metastatic colorectal cancer. Colorectal Dis. 2012;14:31-47.

18. Odom D, Barber B, Bennett L. Health-related quality of life and colorectal cancer-specific symptoms in patients with chemotherapy-refractory metastatic disease treated with panitumumab. Int J Colorectal Dis. 2011;26:173-81.

19. Douillard JY, Siena S, Cassidy J. Randomized, phase III trial of panitumumab with infusional fluorouracil, leucovorin, and oxaliplatin (FOLFOX4) versus FOLFOX4 alone as first-line treatment in patients with previously untreated metastatic colorectal cancer: the PRIME study. J Clin Oncol. 2010;2831:4697-705.

20. Peeters M, Price TJ, Cervantes A. Randomized phase III study of panitumumab with fluorouracil, leucovorin, and irinotecan (FOLFIRI) compared with FOLFIRI alone as second-line treatment in patients with metastatic colorectal cancer. J Clin Oncol. 2010;28:4706-13.

21. Van Cutsem E, Kohne $\mathrm{CH}$, Lang I, et al. Cetuximab plus irinotecan, fluorouracil, and leucovorin as first-line treatment for metastatic colorectal cancer: updated analysis of overall survival according to tumor KRAS and BRAF mutation status. J Clin Oncol. 2011;29:2011-9.

22. Bokemeyer C, Bondarenko I, Hartmann JT, et al. Efficacy according to biomarker status of cetuximab plus FOLFOX-4 as first-line treatment for metastatic colorectal cancer: the OPUS study. Ann Oncol. 2011;22:1535-46.

23. Amado RG, Wolf M, Peeters M, et al. Wild-type KRAS is required for panitumumab efficacy in patients with metastatic colorectal cancer. J Clin Oncol. 2008;26:1626-34.

24. Karapetis CS, Khambata-Ford S, Jonker DJ, et al. K-RAS mutations and benefit from cetuximab in advanced colorectal cancer. $\mathrm{N}$ Engl J Med. 2008;359:1757-65.

25. Van Cutsem E, Lang I, D'haens G, et al. KRAS status and efficacy in the first-line treatment of patients with metastatic colorectal cancer (mCRC) treated with FOLFIRI with or without cetuximab: the CRYSTAL experience. J Clin Oncol. 2008;26:5s.

26. Grothey A. EGFR antibodies in colorectal cancer: where do they belong? J Clin Oncol. 2010;28:4668-70.

27. Instituto Catalán de Oncología. Informe para la Comisión de Farmacia y Terapéutica. Comparativo panitumumab/cetuximabinforme 24-02-11. Disponible en: http://www20.gencat.cat/portal/ site/salut/menuitem.f8d00021b1df07ec3bfd8a10b0c0e1a0/ ?vgnextoid=82b942fc7ea43310VgnVCM2000009b0c1e0aRCRD \&vgnextchannel=82b942fc7ea43310VgnVCM2000009b0c1e0a RCRD\&vgnextfmt=detall\&contentid=e48d9f4e2fe05310Vgn VCM2000009b0c1e0aRCRD. [Fecha de acceso: Junio 2013].

28. Price T, Peeters M, Kim TW, et al. ASPECCT: a randomized, multicenter, open-label, phase 3 study of panitumumab (pmab) vs cetuximab (cmab) for previously treated wild-type (WT) KRAS metastatic colorectal cancer (mCRC). Presented at: European Cancer Congress 2013; September 27-October 1, 2013; Amsterdam, The Netherlands. Abstract LBA18.

29. Erbitux ${ }^{\circledR}$ (cetuximab). Laboratorios Merck. Disponible en: http:// www.ema.europa.eu/docs/es_ES/document_library/EPAR_Product_Information/human/000558/WC500029119.pdf [Fecha de acceso: Junio 2013].

30. Vectibix ${ }^{\circledR}$ (panitumumab). Ficha Técnica. Laboratorios Amgen. Disponible en: http://www.ema.europa.eu/docs/es_ES/document_ library/EPAR_-_Product_Information/human/000741/ WC500047710.pdf [Fecha de acceso: Junio 2013].

31. Gutierrez L, Campos I. Costes de tratamiento con panitumumab y cetuximab en España según las características antropométricas de los pacientes con cáncer colorrectal metastásico. Gac Sanit. 2011;25(EspecCongr 1):71. Disponible en: http://www.aes.es/ 
Jornadas2011/comunicaciones_poster.php (Poster P-58/90). [Fecha de acceso: Junio 2013].

32. http://www.semergen.es/semergen/sessionStart?forwardURL= cda/calculators/calculator.jsp [Fecha de acceso: Junio 2013].

33. Mosteller RD. Simplified calculation of body surface area. N Engl J Med. 1987;22:317.

34. Consejo General del Colegios Oficiales de Farmacéuticos (CGCOF). Disponible en: https://botplusweb.portalfarma.com/ [Fecha de acceso: Junio 2013].

35. Real Decreto Ley 8/2010. BOE de 24 de mayo de 2010. Disponible en: http://www.boe.es/boe/dias/2010/05/24/pdfs/BOE-A2010-8228.pdf [Fecha de acceso: Junio 2013].

36. Gisbert R, Brosa, M. eSalud, base de datos de costes sanitarios en España. 2012. Barcelona: Oblikue Consulting, s.l. Disponible en: http://www.oblikue.com/bddcostes. [Fecha de acceso: Junio 2012].

37. Drummond M, Manca A, Sculpher M. Increasing the generalizability of economic evaluations: recommendations for the design, analysis, and reporting of studies. Int J Technol Assess Health Care. 2005;21:165-71.

38. Claxton K, Sculpher M, McCabe C, et al. Probabilistic sensitivity analysis for NICE technology assessment: not an optional extra. Health Econ. 2005;14:339-47.

39. López Bastida J, Oliva J, Antoñanzas F, et al. Propuesta de guía para la evaluación económica aplicada a las tecnologías sanitarias. Gac Sanit. 2010;24:154-70.

40. Camps C. Minimización de costes en cáncer y sus riesgos. Análisis de la terapia biológica (panitumumab y cetuximab). PharmacoEcon SRA. 2010;7:47-53.
41. Organización Mundial de la Salud. Clasificación internacional del estado nutricional. Disponible en: http://www.euro.who. int/en/what-we-do/health-topics/disease-prevention/nutrition/ahealthy-lifestyle/body-mass-index-bmi [Fecha de acceso: Junio 2013].

42. Antoñanzas-Villar FJ, Oliva J, Velasco M, et al. Costes directos e indirectos del cáncer en España. Cuad Econ ICE. 2006;72:281309.

43. Vieitez JM, Oyagüez I, Casado MA. Análisis de costes del tratamiento del cáncer colorrectal metastásico con bevacizumab y cetuximab. Gac Sanit. 2011;25(EspecCongr 1):80.

44. Zhao Z, Pelletier E, Barber B, et al. Patterns of treatment with chemotherapy and monoclonal antibodies for metastatic colorectal cancer in Western Europe. Curr Med Res Opin. 2012;28:221-9.

45. Moreno V, Martínez-Amores B, Barriuso J, et al. Análisis de coste-efectividad de cetuximab y panitumumab en primera línea para el tratamiento del cáncer colorrectal metastásico en España. Gac Sanit. 2012;26(EspecCongr 1):62.

46. Belda-Iniesta C, Moreno V, Martínez-Amores B, et al. Análisis coste-efectividad basado en predictores de respuesta para terapias de primera línea en cáncer colorrectal metastásico en España. Gac Sanit. 2012;26(EspecCongr 1):61.

47. Sobrero AF, Maurel J, Fehrenbacher L, et al. EPIC: phase III trial of cetuximab plus irinotecan after fluoropyrimidine and oxaliplatin failure in patients with metastatic colorectal cancer. J Clin Oncol. 2008;26:2311-9.

48. Grávalos C, Cassinello J, García-Alfonso P, et al. Integration of panitumumab into the treatment of colorectal cancer. Crit Rev Oncol/Hematol. 2010;74:16-26. 